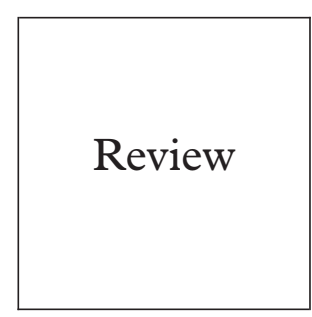

\section{Immunological basis of chlamydia induced reactive arthritis}

\author{
J S H Gaston
}

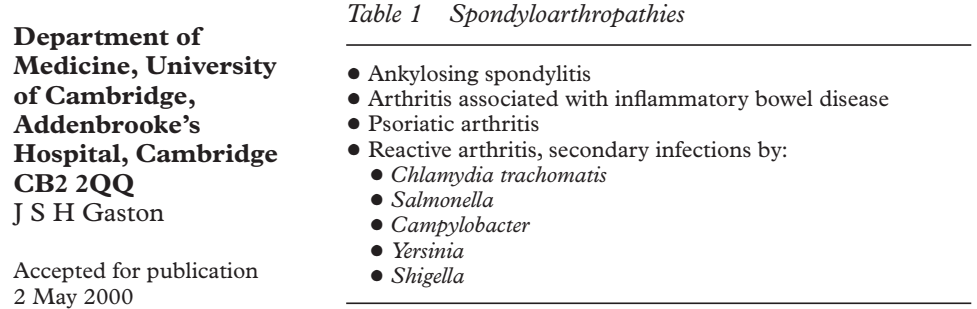

- Ankylosing spondylitis

- Psoriatic arthriti

- Salmonella

- Yersinia

\section{Introduction}

A small proportion of patients who present with clinical symptoms of urethritis or cervicitis later develop inflammatory arthritis and, for an unfortunate minority, this can be the beginning of a persistent and disabling disease. This illness is now termed reactive arthritis, ${ }^{1}$ rather than the older term, Reiter's disease. Reiter described a triad of urethritis, conjunctivitis, and arthritis in first world war soldiers in the trenches following attacks of dysentery, and mistakenly believed that it was due to infection with a novel spirochaete. However, others had previously described the same syndrome, and the triad has no specific pathological significance; patients can have the same arthritis whether they have conjunctivitis or not, and urethritis is not infrequently absent, particularly in cases triggered by gut infection. Because of the emphasis on urethritis in the definition of Reiter's disease, it has often been assumed subsequently that Reiter's disease is synonymous with sexually acquired reactive arthritis (SARA), ${ }^{2}$ whereas in Reiter's report the index cases followed gut infection. For all these reasons the term reactive arthritis is to be preferred and avoids unnecessary categories such as "incomplete" Reiter's disease.

There are many advantages, from a rheumatological view, in studying the pathogenesis of reactive arthritis. Unlike, for instance, rheumatoid arthritis, the onset is sharply defined rather than insidious, and is the result of an identifiable antigenic challenge (in the form of an infectious organism) to the host immune system. It has also been clear for some time that host factors influence who develops arthritis, HLA-B27 being the best described of these, and that the same factors play a part in the whole family of arthritic conditions which make up the seronegative spondyloarthropathies (table 1). There is, therefore, an expectation that exploration of the host:pathogen interaction in reactive arthritis may provide valuable insights into the pathogenesis of related forms of arthritis.

\section{Table 1 Spondyloarthropathies}

\section{Genitourinary infections which trigger reactive arthritis}

Of the sexually acquired infections, the evidence implicating Chlamydia trachomatis as a cause of reactive arthritis is strongest. The infection is not always symptomatic, ${ }^{3}$ and rheumatologists greatly value the help of colleagues in genitourinary medicine in tracking down chlamydial infection in patients with unexplained acute synovitis. There are also reports of the involvement of mycoplasmas, particularly Ureaplasma urealyticum. ${ }^{4}$ These organisms are clearly capable of causing arthritis, and a septic arthritis due to mycoplasma infection in immunodeficiencies in which patients lack antibody is well described. ${ }^{56}$ Individual "reactive arthritis" cases have been reported in which Mycoplasma genitalium, now thought to be a significant cause of nongonococcal, non-chlamydial urethritis, ${ }^{7}$ has been identified in the joint by polymerase chain reaction (PCR) or culture. ${ }^{89}$ Less clear is the extent of mycoplasma infection in classic reactive arthritis. Problems in establishing such an association include the possibility of dual infection by chlamydiae and mycoplasmas, and the carriage of mycoplasmas in the genitourinary tract of normal individuals. As discussed in detail below, finding an organism in joint tissue or fluid, ${ }^{10}$ particularly when using highly sensitive PCR based tests, can no longer be regarded as definitive proof that the organism is responsible for the arthritis.

Infection by gonococcus also gives rise to an inflammatory arthritis which is sometimes loosely referred to as "reactive," although the occasional isolation of the organism by culture has often led to gonococcal arthritis being classified with other forms of septic arthritis. In any case this arthritis does not share the extra-articular features or the HLA-B27 association of classic reactive arthritis, and cannot be regarded as being within the spondyloarthropathy grouping. Again dual gonococcal and chlamydial infection can cause confusion.

\section{Chlamydiae in the joint}

One of the major advances in reactive arthritis research has been the demonstration that triggering organisms such as chlamydiae disseminate from the site of infection to the joint. Atypical elementary bodies were demonstrated in reactive arthritis synovium by immunofluorescence $^{11}$ and electron microscopic studies were also suggestive. ${ }^{12}$ More recently nucleic acid amplification techniques have confirmed the presence of organisms in synovium and 
synovial fluid, ${ }^{13-15}$ and indicated that they are viable since they are transcriptionally active. ${ }^{16}$ Despite this evidence, the organism can rarely if ever be cultured from the joint-the precise status of early reports of cultured chlamydiae remains uncertain. It is also possible to induce a "viable but uncultivable" state in vitro by tryptophan deprivation ${ }^{17} 18$ or treatment with penicillin, ${ }^{19}$ and the chlamydiae may exist in a similar state within macrophages which do not support a productive infection. ${ }^{20}$ Indeed, macrophages or dendritic cells would seem the most likely carriers of organisms into the joint, since they will be able to take up bacteria at the site of infection, ${ }^{21}$ enter the circulation, and be recruited to the synovial membrane. In support of this idea chlamydiae have been detected in peripheral blood leucocytes of reactive arthritis patients ${ }^{22}$ and dendritic cells in the joint stimulate chlamydia specific $\mathrm{T}$ cells. ${ }^{23} \mathrm{It}$ is worth recalling that the synovial membrane is made up of $50 \%$ macrophages (type A synoviocytes), and that recruitment from the blood to the joint occurs normally. It is tempting to speculate that rates of recruitment are higher to larger joints and to those which are subject to low level trauma through weight bearing. These factors might account for the tendency for reactive arthritis preferentially to affect larger joints in the lower limbs. Moreover, the same line of argument leads to the conclusion that macrophages bearing chlamydiae would always be recruited to joints in infected patients, and especially to inflamed joints. Recent evidence supports this; one centre has studied synovial biopsies from a population with a high prevalence of chlamydial infection and showed the presence of organisms in patients with rheumatoid arthritis (RA) or other forms of inflammatory arthritis, where there is a high rate of recruitment of macrophages to a hypertrophic inflamed synovial membrane. $^{2425}$ Chlamydial DNA was even detected in synovium from an asymptomatic joint, although some evidence of low level inflammation was present in this case. ${ }^{26}$ However, the prevalence of detection of chlamydiae in RA synovium was only around $30 \%$ of the prevalence in reactive arthritis.

Recent experimental studies emphasise the capacity of chlamydiae to disseminate; thus chlamydiae were identified in joints of mice experimentally infected in the eye, ${ }^{27}$ and introduction of chlamydiae to one mucosal site has been reported to result in colonisation of others. ${ }^{28}$ This property of $C$ trachomatis is shared by $C$ pneumoniae since it is believed that macrophages infected in the lung or upper respiratory tract deliver organisms to atheromatous plaques in arteries where they may have a role in coronary artery and cerebrovascular disease..$^{29} 30$

The findings in reactive arthritis induced by chlamydia infection are mirrored by those in reactive arthritis triggered by gut infection, where organisms have been demonstrated in synovium and synovial fluid by immunofluorescence, mainly associated with phagocytes. $^{31}{ }^{32}$ Initial results using PCR to detect nucleic acids were negative, suggesting a differ- ence between chlamydia induced and enteric reactive arthritis, but very recent observations have shown transcriptionally active yersiniae in a reactive arthritis joint. ${ }^{33}$ In the case of enteric infections, there is evidence to suggest persistence of the infection long after the symptomatic gut infection has resolved-yersinia antigens have been detected in arthritic joints months to years after infection. The same may apply to chlamydial infection, and it will be important to identify sites of persistence and the extent to which the organism is susceptible to treatment with antibiotics while in a quiescent state.

\section{The immune response to chlamydiae in the joint}

T CELL MEDIATED IMMUNE RESPONSES

Chlamydiae are obligate intracellular pathogens and as such require $\mathrm{T}$ cell mediated immunity to control the infection. ${ }^{34}$ Antibody may have a role in preventing reinfection, especially when it is produced locally in the genital tract, but in most circumstances has little if any role in clearing the organism. ${ }^{35}$ It is not surprising therefore that $\mathrm{T}$ cell mediated immune responses have been readily demonstrated in the joints of patients with chlamydial induced reactive arthritis. ${ }^{36}$ Indeed, characterising this immune response has contributed substantially to what is known about cell mediated responses to $C$ trachomatis in humans, with the identification of several of the antigenic components of chlamydiae which elicit $\mathrm{T}$ cell mediated responses. ${ }^{37}{ }^{38} \mathrm{CD} 4+\mathrm{T}$ cells play the major part in controlling chlamydial infection, ${ }^{39}$ probably through their production on interferon $\gamma,{ }^{40}$ but protective CD8+ T cells have also been described in mice, ${ }^{41}$ and more recently in humans. ${ }^{42}$ The relative importance of responses by these subsets in humans has not yet been established, and chlamydia specific CD8+ $\mathrm{T}$ cells have not yet been isolated from human joints. Nevertheless, $\mathrm{CD} 8+\mathrm{T}$ cells are activated in the joint and also produce a similar set of cytokines as CD4+ T cells (H Beacock-Sharp, J S H Gaston, unpublished data), and the failure to isolate chlamydia specific $T$ cells may simply reflect technical difficulties in working with these cells.

\section{IMMUNE RESPONSES TO HSP60}

Among the antigens commonly recognised is the chlamydial $60 \mathrm{kDa}$ heat shock protein (hsp60). All bacteria, indeed all living cells, need to express hsp60 as part of their normal functioning-it is used to ensure cellular proteins are correctly folded after synthesis. Recent data point to the possibility that, in addition to a physiological role in protein folding, extracellular hsp60 may be recognised by myeloid cells, resulting in the production of pro-inflammatory cytokines, in a manner similar to lipopolysaccharide. ${ }^{43}$ This effect has been shown for chlamydial hsp60. ${ }^{44} 45$

However, immune recognition of hsp60 is also very common in infection, particularly where intracellular organisms are concerned. ${ }^{46}$ Considerable attention has been paid to the possibility that immune responses to hsp60 are 
Table 2 Evidence implicating immune responses to Chlamydia hsp60 in inflammation

- Raised anti-hsp60 antibodies in patients with tubal infertility

- DTH responses to hsp60 in primed animal, in models of trachoma and salpingitis

- Continued production of hsp60 by chlamydiae in a non-cultivable state

- Transcription of hsp60 genes by synovial chlamydiae

important in chlamydia induced pathology. There are reasons for this, detailed in table 2 . Studies of $\mathrm{T}$ cell recognition of hsp60 in reactive arthritis have produced two major findings. Firstly, although it has been postulated that T cells reactive to chlamydial hsp60 might cross react with the human counterpart (since they have highly conserved amino acid sequences), precise mapping of the peptides (epitopes) within chlamydial hsp60 which are recognised by CD4+ T cells has so far not shown any evidence of cross reactivity with human hsp60. Secondly, in two instances the epitope identified in $C$ trachomatis hsp60 was identical or nearly identical in $C$ pneumoniae hsp $60^{47}$ and $\mathrm{R}$ Raggiaschi, J S H Gaston, unpublished data. Since infection with $C$ pneumoniae is common, particularly in early life, many of those infected for the first time with $C$ trachomatis will already have immune systems primed to respond to chlamydial hsp60 because of previous encounter with $C$ pneumoniae. The idea that priming might be important is a recurring theme in consideration of chlamydia induced pathology. Infection of the conjunctiva which leads to scarring trachoma requires recurrent infection, ${ }^{48}$ and in an animal model of this condition, disease was produced by challenging primed animals with hsp60..$^{49}$ Infertility is also related to recurrent infection. ${ }^{50}$ In reactive arthritis there is rarely a history of previous symptomatic genitourinary infection, but infection by $C$ pneumoniae, which mostly causes mild upper respiratory tract infections, could be a sufficient priming event. This idea is currently under further investigation.

CYTOKINE PRODUCTION IN THE REACTIVE ARTHRITIS JOINT

If $\mathrm{T}$ lymphocytes encounter bacterial antigen in the joint they will be activated to produce the cytokines which ultimately control joint inflammation and destruction. Synovial T lymphocytes from reactive arthritis joints analysed ex vivo show spontaneous production of interferon $\gamma$ (H Beacock-Sharp, J S H Gaston, unpublished data), generally regarded as a proinflammatory cytokine, and also the critical factor in overcoming chlamydial infection. Although all reports agree on the presence of interferon $\gamma$, it has been claimed that T cells making interleukin 4 (IL-4) are also present in the joint - in contrast with rheumatoid arthritis where IL-4 is clearly absent. ${ }^{51}$ This is relevant to pathogenesis, using the model which classifies $\mathrm{T}$ cells into two categories according to their pattern of cytokine production-Th1 cells dominated by interferon $\gamma$ and Th2 cells dominated by IL-4, the two subsets of T cells being mutually antagonistic. ${ }^{52}$ Since immunity to chlamydiae requires a Th1 response, it has been argued that the presence of IL- 4 could indicate an inadequate Th1 type response to chlamydiae which could lead to persistence of the organism in the joint. Studies in mice lacking the interferon $\gamma$ gene indicate that apart from an inability to clear chlamydial infection, such mice may sustain a vigorous and potentially tissue damaging delayed type hypersensitivity responses driven by $\mathrm{Th} 2$ cytokines, particularly IL-4. IL-10 is another cytokine which antagonises Th1 responses, and this is certainly present in the joint, but whether the amounts are appropriate is not known. In animal models of chlamydial infection, clearance of the organism is affected by the relative balance between interferon $\gamma$ and IL-10 production, ${ }^{53}$ and IL-10 gene knockout mice clear chlamydial infection more rapidly than normal. ${ }^{54}$ One of the most interesting $\mathrm{T}$ cell derived cytokines which is likely to be relevant to joint destruction is the recently described IL-17, ${ }^{55}$ which is present in reactive arthritis joints. IL-17 can mimic many of the properties of IL- 1 and tumour necrosis factor $\alpha$ (TNF $\alpha)$ which lead to cartilage breakdown and bone erosion. $^{56-58}$

In addition to cytokines derived from $T$ cells, and those whose production is induced by $\mathrm{T}$ cells, proinflammatory cytokines may also be produced by cells in the synovium as a result of infection itself. The cells include both macrophage-like synoviocytes which can make IL-1 and TNF $\alpha$ and fibroblast-like synoviocytes which also have the ability to make cytokines. ${ }^{59}$ These effects of infection in the joint may parallel those at the initial site of infection, where chlamydiae induce cytokine production by epithelial cells. ${ }^{60}$

SUSCEPTIBILITY TO REACTIVE ARTHRITIS

Only a small minority of patients infected with chlamydiae develops arthritis. One factor influencing susceptibility is HLA-B27, but it is important not to overstate its influence. In studies of reactive arthritis triggered by enteric infection (such as an outbreak of salmonella food poisoning), only about $30 \%$ of patients are $\mathrm{B} 27+$ if care is taken to include all patients who develop symptoms, and not just the subsection who have disease severe enough to require hospital referral. ${ }^{61}$ The prevalence of HLA-B27 in the latter group is much higher, $60-80 \%$, so B27 seems to influence severity and persistence rather than just susceptibility. If the rate of recruitment of macrophages containing chlamydiae is an important factor, patients with some level of pre-existing inflammation in joints - for example, as a result of low grade trauma, may be more susceptible. Many affected patients are young and athletic, and not infrequently reactive arthritis is first attributed to a sporting injury. Other relevant factors may include previous exposure to chlamydial antigens, and the quality of the immune response which they elicit in particular individuals. The latter can be influenced by genetic factors such as polymorphisms in cytokine genes which alter rates of production. Such factors may be identified by whole genome screening. This is impractical in reactive arthritis but is being applied to ankylosing spondylitis. ${ }^{62}$ Genes relevant to susceptibility to 
this disease might well also contribute to susceptibility to reactive arthritis.

\section{Why does reactive arthritis sometimes persist?}

There is a subtle problem with the question of persistence in reactive arthritis. Those with persistent arthropathy are those most likely to be HLA-B27+ and these patients in turn are those most susceptible to other forms of spondyloarthropathy. Thus, it can be argued that reactive arthritis does not persist or evolve into something resembling ankylosing spondylitis; instead the patients develop two B27 associated conditions with overlapping courses. Although this may happen, and patients with longstanding ankylosing spondylitis are seen who develop an episode of acute reactive arthritis, the more usual clinical picture is one of evolution from typical reactive arthritis to chronic spondyloarthropathy. This impression is strengthened by the investigations showing evidence of persistence of the triggering organism or its antigens in the affected joint years after the initial infection. Therefore, persistence is real and not an ascertainment artefact in the B27+ disease susceptible population.

To discuss reasons for persistence of arthritis it is reasonable to start with inquiry into the mechanisms which normally bring about resolution of joint inflammation in reactive arthritis. If bacterial antigens are critical to its pathogenesis, resolution may simply represent the final clearance of the organism from the joint, or from another site of persistent infection so that no more chlamydiae are delivered to the synovium. In that case persistence could relate to a failure to clear the organism, if there was persistent infection in, for instance, the prostate, chlamydial antigens would continue to be delivered to the joint. Evidence from enteric reactive arthritis now strongly suggests that this process can occur for months or even years, and as noted already, chlamydiae have also been identified in reactive arthritis synovium in persistent reactive arthritis-that is, years after the initial triggering infection, although it is difficult to exclude reinfection. Among the genes which chlamydiae have been shown to transcribe in the joint is hsp60, ${ }^{16}$ implying production of this potent stimulus to the immune system. Alternatively, if the main immune response in persistent arthritis is no longer directed against bacterial antigens but against something normally expressed in the joint (an autoantigen), arthritis would persist even when infection had been entirely eradicated. As previously discussed, autoimmune responses have not been identified in reactive arthritis, but the possibility is still worth pursuing.

However, even in situations where antigen persists, the immune system has mechanisms for downregulating immune responses appropriately so that inflammation is not sustained unnecessarily. These include the death of effector cells by apoptosis and the production of anti-inflammatory cytokines such as IL-4, IL-10, IL-13, and transforming growth factor $\beta$
(TGF $\beta$ ). Any one of these might fail in persistent reactive arthritis.

\section{Treatment}

If arthritis relates to persistent infection by reactive arthritis associated organisms, it is reasonable to ask whether antibiotics have any part to play in the treatment of the disease. Although infection in the joint has been demonstrated, this is at a very low level, and the assumption is that some other reservoir of infection allows continuous colonisation of the synovial membrane. The same idea underlies other forms of chronic inflammation associated with both $C$ trachomatis and $C$ pneumoniae. In the latter case, the hypothesis that the presence of the organism within atherosclerotic plaques accelerates cardiovascular disease has led to several trials of long term antibiotics (including the macrolide azithromycin) with some intriguing preliminary results which require confirmation in much larger trials. ${ }^{63}$ In reactive arthritis evidence is conflicting but generally fails to support a role for antibiotics; trials using ciprofloxacin in yersinia induced reactive arthritis have been negative. ${ }^{64}{ }^{65}$ However, one study showed an effect of treating chlamydial induced reactive arthritis with long term tetracycline, though this result depended on subgroup analysis. ${ }^{66} \mathrm{~A}$ further trial of azithromycin has just been completed but results are not yet available. One problem with the use of antibiotics is the assumption that conventional treatment regimens will be effective against organisms which are dividing very slowly, if at all, and which may be able to persist intracellularly. Recent results in a rat model of yersinia induced arthritis underlined this, since rats continued to excrete yersinia organisms and had no relief from arthritis despite prolonged treatment with ciprofloxacin. ${ }^{67}$ It has even be suggested that antibiotics could even exacerbate late arthritis by driving organisms into a persistent state. Thus, further information about the sites of persistence and the state of the organism will be required to devise and test rational antibiotic regimens.

An alternative view of the pathogenesis of the reactive arthritis is that it is the nature of the immune response to bacterial antigens which governs the development of arthritis and its persistence. Thus an immune response to a bacterial antigen might cross react with a self protein, as postulated in Lyme disease ${ }^{68}$ or the immune response might be excessive as postulated in tuberculous leprosy, with concomitant inflammation and tissue damage. Under these circumstances therapy would be required to modulate the immune response-for example, by reintroducing tolerance to the self antigen (while preserving the immune response to other bacterial antigens), or by altering the nature of the immune response to become less pro-inflammatory with less production of interferon $\gamma$ and $\mathrm{TNF} \alpha$ and a greater production of IL-4, IL-10, or TGF $\beta$. Yet another view is that the $\mathrm{T}$ cell response is inadequate and fails to clear the infection satisfactorily, and if this were true, therapy would be directed towards boosting the immune response. Since 


\section{Summary}

- Chlamydial infection can trigger reactive arthritis in susceptible individuals

- The joint contains viable organisms and a T cell mediated immune response to chlamydial antigens

- Possible pathogenic mechanisms include:

- Proinflammatory immune responses to persistent chlamydia organisms/antigens

- Development of an autoimmune response stimulated by a bacterial antigen

- Failure to clear chlamydial infection

- One or more of these mechanisms may be at work in different patients (self limiting or chronic arthritis) or at different times in the course of arthritis (early, late)

- Until pathogenesis is clarified, treatment is empirical - conventional courses of antibiotic, symptomatic measures for arthritis, and in severer cases some of the disease modifying drugs which are effective in other forms of chronic inflammatory arthritis (sulphasalazine, methotrexate).
17 Beatty WL, Belanger TA, Desai AA, et al. Tryptophan depletion as a mechanism of gamma interferon-mediate chlamydial persistence. Infect Immun 1994;62:3705-11.

18 Beatty WL, Morrison RP, Byrne GI. Persistent chlamydiae: from cell culture to a paradigm for chlamydial pathogenesis. Microbiol Rev 1994;58:686-99.

19 Chopra I, Storey C, Falla TJ, et al. Antibiotics, peptidoglycan synthesis and genomics: the chlamydial anomaly revisited. Microbiology 1998;144(Pt 10):2673-8.

20 Gerard HC, Kohler L, Branigan PJ, et al. Viability and gene expression in Chlamydia trachomatis during persistent infection of cultured human monocytes. Med Microbiol Immunol 1998;187:115-20.

21 Stagg AJ, Tuffrey M, Woods C, et al. Protection against ascending infection of the genital tract by Chlamydia trachomatis is associated with recruitment of major histocompatibility complex class II antigen-presenting cells into uterine tissue. Infect Immun 1998;66:3535-44.

22 Kuipers JG, Jurgenssaathoff B, Bialowons A, et al. Detection of Chlamydia trachomatis in peripheral blood leukocytes of reactive arthritis patients by polymerase chain reaction. Arthritis Rheum 1998;41:1894-5.

23 Stagg AJ, Hughes RA, Keat AC, et al. Antigen-presenting cells but not lymphocytes in the joint may indicate the cause of reactive arthritis. Br f Rheumatol 1996;35:108290 .

24 Schumacher H, Arayssi T, Branigan P, et al. Surveying for evidence of synovial Chlamydia trachomatis by polymerase chain reaction (PCR). A study of 411 synovial biopsies and synovial fluids. Arthritis Rheum 1997;40:S270.

25 Schumacher HR, Gerard HC, Arayssi TK, et al. Lower prevalence of Chlamydia pneumoniae DNA compared with Chlamydia trachomatis DNA in synovial tissue of arthritis patients. Arthritis Rheum 1999;42:1889-93.

this would mean trying to increase levels of interferon $\gamma$ and $\mathrm{TNF} \alpha$ (these cytokines being the most effective against intracellular pathogens), the therapeutic approach would be diametrically opposed to that suggested above where the immune response is considered to be the culprit in bringing about joint inflammation, and consequently in need of downregulation. Clearly it is important to know which model of arthritis pathogenesis is correct in order to propose rational therapy.

1 Gaston J. Symposium: reactive arthritis. Rheumatology in Europe 1995;24:5-22

2 Keat A, Maini R, Nkwazi G, et al. Role of Chlamydia trachomatis and HLA-B27 in sexually acquired reactive arthritis. $B M \mathcal{F} 1978 ; 1: 605$

3 Wollenhaupt J, Kolbus F, Weissbrodt $\mathrm{H}$, et al. Manifestations of Chlamydia induced arthritis in patients with silen versus symptomatic urogenital chlamydial infection. Clin Exp Rheumatol 1995;13:453-8.

4 Horowitz S, Horowitz J, Taylor-Robinson D, et al. Ureaplasma urealyticum in Reiter's syndrome. $\mathcal{F}$ Rheumatol 1994;21:877-82.

5 Webster AD, Taylor-Robinson D, Furr PM, et al. Mycoplasmal (ureaplasma) septic arthritis in hypogammaglobulinaemia. BMf 1978;1:478-9.

6 Furr PM, Taylor-Robinson D, Webster AD. Mycoplasmas and ureaplasmas in patients with hypogammaglobulinaemia and their role in arthritis: microbiological observ

7 Maeda S, Tamaki M, Nakano M, et al. Detection of Mycoplasma genitalium in patients with urethritis. $\mathcal{F}$ Urol 1998;159:405-7.

8 Taylor-Robinson D, Gilroy CB, Horowitz S, et al. Mycoplasma genitalium in the joints of two patients with arthritis. Eur f Clin Microbiol Infect Dis 1994;13:1066-9.

9 Tully JG, Rose DL, Baseman JB, et al. Mycoplasma pneumoniae and Mycoplasma genitalium mixture in synovial fluid isolate. $\mathcal{F}$ Clin Microbiol 1995;33:1851-5.

10 Schaeverbeke T, Gilroy CB, Bebear C, et al. Mycoplasma fermentans in joints of patients with rheumatoid arthritis and other joint disorders. Lancet 1996;347:1418.

11 Keat A, Thomas B, Dixey J, et al. Chlamydia trachomatis and reactive arthritis - the missing link. Lancet 1987;i:7274 .

12 Schumacher HR, Magge S, Cherian PV, et al. Light and electron microscope studies on the synovial membrane in Reiter's syndrome. Arthritis Rheum 1988:31:937-46.

13 Taylor-Robinson D, Gilroy C, Thomas B, et al. Detection of Chlamydia trachomatis DNA in joints of reactive arthritis patients by polymerase chain reaction. Lancet 1992;340 81-2.

14 Rahman MU, Cheema MA, Schumacher HR, et al. Molecular evidence for the presence of Chlamydia in the synovium of patients with Reiter's syndrome. Arthritis Rheum 1992;35:521-9.

15 Branigan PJ, Gerard HC, Hudson AP, et al. Comparison of synovial tissue and synovial fluid as the source of nucleic acids for detection of Chlamydia trachomatis by polymerase chain reaction. Arthritis Rheum 1996;39:1740-6.

16 Gerard HC, Branigan PJ, Schumacher HR, et al. Synovial Chlamydia trachomatis in patients with reactive arthritis/ Chlamydia trachomatis in patients with reactive arthritis/
Reiter's syndrome are viable but show aberrant gene expression. F Rheumatol 1998;25:734-42.
26 Schumacher HR, Arayssi T, Crane M, et al. Chlamydia trachomatis nucleic acids can be found in the synovium of some asymptomatic subjects. Arthritis Rheum 1999;42: $1281-4$

27 Whittum-Hudson J, Gerard H, Branigan P, et al. Presence and pathogenesis of Chlamydia trachomatis in synovium after ocular infection in mice. Arthritis Rheum 1997;40: S143

28 Perry LL, Hughes S. Chlamydial colonization of multiple mucosae following infection by any mucosal route. Infect Imтии 1999;67:3686-9.

29 Thom D, Grayston J, Siscovick D, et al. Association of prior infection with Chlamydia pneumoniae and angiographically demonstrated coronary artery disease. $7 A M A 1992$; 268:68-72.

30 Melnick S, Sharar E, Folsom A, et al. Past infection with Chlamydia pneumoniae strain TWAR and asymptomatic carotid atherosclerosis. Am 7 Med 1993;95:499-504.

31 Granfors K, Jalkanen S, von Essen R, et al. Yersinia antigens in synovial fluid cells from patients with reactive arthritis. $N$ Engl f Med 1989;320:216-21.

32 Granfors K, Jalkanen S, Lindberg A, et al. Salmonella reactive arthritis. Lancet 1990;335:685-8. evidence for persistent Yersinia infection in reactive arthritis. Arthritis Rheum 1999;42:2239-42.

34 Yang X, Hayglass KT, Brunham RC. Different roles are played by alpha beta and gamma delta $T$ cells in acquired immunity to Chlamydia trachomatis pulmonary infection. Immunology 1998;94:469-75.

35 Ramsey K, Soderberg L, Rank R. Resolution of chlamydial genital infection in B-cell-deficient mice and immunity to reinfection. Infect Immun 1988;56:1320-5.

36 Gaston JSH, Life PF, MerilahtiPalo R, et al. Synovial T lymphocyte recognition of organisms that trigger reactive arthritis. Clin Exp Immunol 1989;76:348-53.

37 Hassell AB, Reynolds DJ, Deacon M, et al. Identification of T-cell stimulatory antigens of Chlamydia trachomatis using 513-19.

38 Gaston JSH, Deane KHO, Jecock RM, et al. Identification of 2 Chlamydia trachomatis antigens recognized by synovial fluid $\mathrm{T}$ cells from patients with Chlamydia induced reactive arthritis. F Rheumatol 1996;23:130-6.

39 Su H, Caldwell HD. CD4(+) T cells play a significant infection of the mouse genital tract. Infect Immun 1995;63:
3302-8.

40 Cotter TW, Ramsey KH, Miranpuri GS, et al. Dissemination of Chlamydia trachomatis chronic genital tract infection in gamma interferon gene knockout mice. Infect Immun 1997;65:2145-52.

41 Starnbach MN, Bevan MJ, Lampe MF. Murine cytotoxic T lymphocytes induced following Chlamydia trachomatis intraperitoneal or genital tract infection respond to cells infected with multiple serovars. Infect Immun 1995;63: 3527-30.

$42 \mathrm{Kim}$ SK, Angevine $\mathrm{M}$, Demick $\mathrm{K}$, et al. Induction of HLA class I-restricted CD8(+) CTLs specific for the major outer membrane protein of Chlamydia trachomatis in human genital tract infections. F Immunol 1999;162: 6855-66.

43 Chen W, Syldath U, Bellmann K, et al. Human 60-kDa heat-shock protein: a danger signal to the innate immune system. F Immunol 1999;162:3212-9.

$44 \mathrm{Kol} \mathrm{A}$, Bourcier T, Lichtman AH, et al. Chlamydial and human heat shock protein 60 s activate human vascular
endothelium, smooth muscle cells, and macrophages. $\mathcal{F}$ Clin Invest 1999;103:571-7. lipopolysaccharide in synovial cells from patients with

33 Gaston JSH, Cox C, Granfors K. Clinical and experimental synovial fluid-derived T-cell clones. Immunology 1993;79: role in adoptive immunity to Chlamydia trachomatis 
$45 \mathrm{Kol} \mathrm{A}$, Lichtman AH, Finberg RW, et al. Cutting edge: heat shock protein (HSP) 60 activates the innate immune response: CD14 is an essential receptor for HSP60 activa

46 tion of mononuclear cells. F Immunol 2000;164:13-7. Gaston JSH. Heat shock proteins and arthr
readers start here. Autoimmunity 1997;26:33-42.

47 Deane K, Jecock R, Pearce J, et al. Identification and characterization of a DR4-restricted T cell epitope within chlamydia hsp60. Clin Exp Immunol 1997;109:439-45.

48 Bobo LD, Novak N, Munoz B, et al. Severe disease in children with trachoma is associated with persistent infection. F Infect Dis 1997;176:1524-30.

49 Morrison R, Belland R, Lyng K, et al. Chlamydial disease pathogenesis. The $57 \mathrm{kD}$ chlamydial hypersensitivity antigen is a stress response protein. 7 Exp Med 1989;170: $1271-83$.

50 Vanvoorhis WC, Barret LK, Sweeney YTC, et al. Repeated Chlamydia trachomatis infection of Macaca nemestrina fallopian tubes produces a Th1-like cytokine response associated with fibrosis and scarring. Infect Immun 1997;65: ated with

51 Simon AK, Seipelt E, Sieper J. Divergent T-cell cytokine patterns in inflammatory arthritis. Proc Natl Acad Sci USA 1994;91:8562-6.

52 Mosmann T, Sad S. The expanding universe of T-cell subsets: Th1, Th2 and more. Immunol Today 1996;17:13846.

53 Kotake S, Schumacher HR, Arayssi TK, et al. Gamma interferon and interleukin-10 gene expression in synovial tissues from patients with early stages of Chlamydiaassociated arthritis and undifferentiated oligoarthritis and from healthy volunteers. Infect Immun 1999;67:2682-6.

54 Yang X, Gartner J, Zhu LH, et al. IL-10 gene knockout mice show enhanced Th1-like protective immunity and absent
granuloma formation following Chlamydia trachomatis lung infection. F Immunol 1999;162:1010-17.

55 Yao ZB, Painter SL, Fanslow WC, et al. Human IL-17: a novel cytokine derived from T cells. F Immunol 1995; 155 : 5483-6.

56 Lotz M, Bober L, Narula S, et al. IL-17 promotes cartilage degradation. Arthritis Rheum 1996;39:S120.

57 Kotake S, Udagawa N, Takahashi N, et al. IL-17 in synovial fluids from patients with rheumatoid arthritis is a poten stimulator of osteoclastogenesis. F Clin Invest 1999;103: $1345-52$
58 Aarvak T, Chabaud M, Miossec P, et al. IL-17 is produced by some proinflammatory Th1/Th0 cells but not by Th2 cells. F Immunol 1999;162:1246-51.

59 Rodel J, Straube E, Lungershausen W, et al. Secretion of cytokines by human synoviocytes during in vitro infection with Chlamydia trachomatis. If Rheumatol 1998;25: 2161-8.

60 Rasmussen SJ, Eckmann L, Quayle AJ, et al. Secretion of proinflammatory cytokines by epithelial cells in response to Chlamydia infection suggests a central role for epithelial cells in chlamydial pathogenesis. F Clin Invest 1997;99:7787.

61 Leirisalo-Repo M. Prognosis, course of disease, and treatment of the spondyloarthropathies. Rheum Dis Clin North Am 1998;24:737-51.

62 Brown M, Kennedy L, MacGregor A, et al. Genetic susceptibility to ankylosing spondylitis in twins: the role of genes, HLA and the environment. Arthritis Rheum 1997;41:58895.

63 Grayston JT. Design of future intervention studies for Chlamydia pneumoniae in atherosclerosis. Am Heart $\mathcal{f}$ 1999;138(5 Pt 2):S556-7.

64 Sieper J, Fendler C, Laitko S, et al. No benefit of long-term ciprofloxacin treatment in patients with reactive arthritis and undifferentiated oligoarthritis - a three-month, multicenter, double-blind, randomized, placebo-controlled study. Arthritis Rheum 1999;42:1386-96.

65 Wakefield D, McCluskey P, Verma M, et al. Ciprofloxacin treatment does not influence course or relapse rate of reactive arthritis and anterior uveitis. Arthritis Rheum 1999;42: 1894-7.

66 Lauhio A, LeirisaloRepo M, Lahdevirta J, et al. Doubleblind, placebo-controlled study of three-month treatment with lymecycline in reactive arthritis with special reference to chlamydia arthritis. Arthritis Rheum 1991;34:6-14.

67 Zhang Y, Gripenberglerche C, Soderstrom KO, et al. Antibiotic prophylaxis and treatment of reactive arthritis: lessons from an animal model. Arthritis Rheum 1996;39: 1238-43.

68 Gross DM, Forsthuber T, Tarylehmann M, et al. Identification of LFA-1 as a candidate autoantigen in treatmentresistant Lyme arthritis. Science 1998;281:703-6. 